\title{
L'homologie de Novikov des entrelacs de Waldhausen
}

\author{
David Cimasoni \\ Section de mathématiques, Université de Genève, 2-4 rue du Lièvre, 1211 Genève 24, Suisse \\ Courriel :David.Cimasoni@ math.unige.ch
}

(Reçu le 2 octobre 2001, accepté le 8 octobre 2001)

Résumé. Un multi-entrelacs de Waldhausen est un entrelacs avec multiplicités dans une 3-sphère d'homologie dont l'extérieur est une variété de Waldhausen. Dans cette Note, nous calculons l'homologie de Novikov des multi-entrelacs de Waldhausen. En corollaire, nous obtenons une borne pour le nombre de modules de Novikov d'un entrelacs de Waldhausen donné. (c) 2001 Académie des sciences/Éditions scientifiques et médicales Elsevier SAS

\section{The Novikov homology of graph links}

Abstract. A graph multilink is a link with multiplicities in a homology 3-sphere whose exterior is a graph manifold. In this Note, we compute the Novikov homology of graph multilinks. As a corollary, we give a majoration for the number of Novikov modules on a given graph link. (C) 2001 Académie des sciences/Éditions scientifiques et médicales Elsevier SAS

\section{Introduction}

Soit $L=L_{1} \cup \cdots \cup L_{n}$ un entrelacs orienté dans une 3-sphère d'homologie $\Sigma$. Considérons son extérieur $X=\Sigma-\operatorname{int} \mathcal{N}(L)$, où $\mathcal{N}(L)$ est un voisinage tubulaire de $L$. Les revêtements infini cycliques de $X$ sont classifiés par

$$
\left[X, S^{1}\right] \simeq \operatorname{Hom}\left(\pi_{1} X, \mathbb{Z}\right)=\operatorname{Hom}\left(H_{1} X, \mathbb{Z}\right) \simeq H^{1} X \simeq H_{1} L=\bigoplus_{i=1}^{n} \mathbb{Z}\left[L_{i}\right] .
$$

Ainsi, chaque revêtement infini cyclique de $X$ correspond à des multiplicités $\underline{m}=\left(m_{1}, \ldots, m_{n}\right)$, où $m_{i}$ est un entier associé à $L_{i}$. Un entrelacs orienté $L$ muni d'une telle suite de multiplicités $\underline{m}$ est appelé un multi-entrelacs, et noté $L(\underline{m})=m_{1} L_{1} \cup \cdots \cup m_{n} L_{n}$.

Si $\widetilde{X}(\underline{m}) \stackrel{p}{\rightarrow} X$ désigne le revêtement déterminé par $\underline{m}$, le groupe abélien $H_{1}(\widetilde{X}(\underline{m}))$ est muni d'une structure naturelle de $\mathbb{Z}\left[t, t^{-1}\right]$-module: on parle du module d'Alexander de $L(\underline{m})$. Comme il existe toujours une matrice de présentation carrée de ce module, le polynôme d'Alexander de $L(\underline{m})$ peut être défini comme le déterminant d'une telle matrice.

L'homologie de Novikov de $L(\underline{m})$ est le $\mathbb{Z} \llbracket t \rrbracket\left[t^{-1}\right]$-module $\widehat{H}_{L}(\underline{m})=H_{1}(\tilde{X}(\underline{m})) \otimes_{\mathbb{Z}\left[t, t^{-1}\right]} \mathbb{Z} \llbracket t \rrbracket\left[t^{-1}\right]$. Cette homologie, introduite par S.P. Novikov en 1981, lui permit de construire un analogue de la théorie de Morse pour les 1-formes fermées non-exactes.

Note présentée par Étienne GHYS

S0764-4442(00)02159-0/FLA

(C) 2001 Académie des sciences/Éditions scientifiques et médicales Elsevier SAS. Tous droits réservés. 


\section{David Cimasoni}
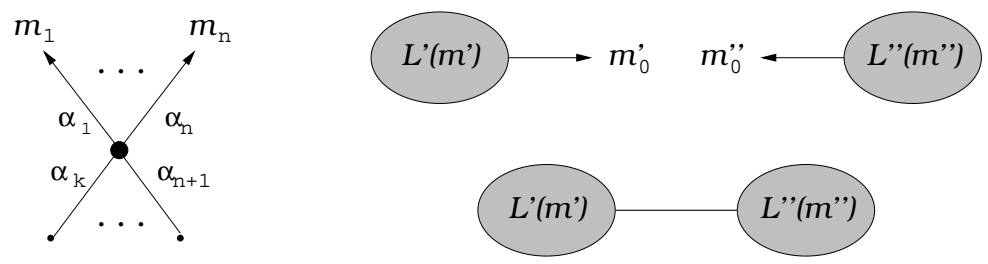

Figure 1: Diagrammes d'épissure/Splice diagrams

Exemple 1. - Un multi-entrelacs $L(\underline{m})$ est dit fibré s'il existe une fibration localement triviale $X \stackrel{\phi}{\rightarrow} S^{1}$ dans la classe d'homotopie $\underline{m}$. Le polynôme d'Alexander d'un tel multi-entrelacs est une unité de l'anneau $\mathbb{Z} \llbracket t \rrbracket\left[t^{-1}\right]$; l'homologie de Novikov est donc triviale.

Voici une variation d'un problème posé par Novikov dans [4]: étant donné un entrelacs $L=L_{1} \cup \cdots \cup L_{n}$ dans une 3-sphère d'homologie, comment se comporte l'homologie de Novikov $\widehat{H}_{L}(\underline{m})$ en fonction de $\underline{m} \in \mathbb{Z}^{n}$ ? Nous donnons une réponse complète pour les entrelacs de Waldhausen, qu'il s'agit à présent de définir.

\section{Entrelacs de Waldhausen}

Soient $L^{\prime}=L_{0}^{\prime} \cup L_{1}^{\prime} \cup \cdots \cup L_{n}^{\prime}$ et $L^{\prime \prime}=L_{0}^{\prime \prime} \cup L_{1}^{\prime \prime} \cup \cdots \cup L_{r}^{\prime \prime}$ deux entrelacs d'extérieurs $X^{\prime}$ et $X^{\prime \prime}$ dans des sphères d'homologie $\Sigma^{\prime}$ et $\Sigma^{\prime \prime}$. Choisissons des voisinages tubulaires $\mathcal{N}\left(L_{0}^{\prime}\right)$ et $\mathcal{N}\left(L_{0}^{\prime \prime}\right)$ munis de parallèles et de méridiens standards $P^{\prime}, M^{\prime} \subset \partial \mathcal{N}\left(L_{0}^{\prime}\right)$, et $P^{\prime \prime}, M^{\prime \prime} \subset \partial \mathcal{N}\left(L_{0}^{\prime \prime}\right)$; posons $\Sigma=\left(\Sigma^{\prime}-\mathcal{N}\left(L_{0}^{\prime}\right)\right) \cup_{h}\left(\Sigma^{\prime \prime}-\right.$ $\left.\mathcal{N}\left(L_{0}^{\prime \prime}\right)\right)$, où $h: \partial \mathcal{N}\left(L_{0}^{\prime}\right) \rightarrow \partial \mathcal{N}\left(L_{0}^{\prime \prime}\right)$ est un homéomorphisme envoyant $P^{\prime}$ sur $M^{\prime \prime}$ et $M^{\prime}$ sur $P^{\prime \prime}$. Il est facile de vérifier que $\Sigma$ est une sphère d'homologie. L'entrelacs $L=L_{1}^{\prime} \cup \cdots \cup L_{n}^{\prime} \cup L_{1}^{\prime \prime} \cup \cdots \cup L_{r}^{\prime \prime}$ dans $\Sigma$ est appelé l'épissure de $L^{\prime}$ et $L^{\prime \prime}$ le long de $L_{0}^{\prime}$ et $L_{0}^{\prime \prime}$; il est noté

$$
L=L^{\prime} \overline{L_{0}^{\prime} \quad L_{0}^{\prime \prime}} L^{\prime \prime} .
$$

L'épissure $L^{\prime}\left(\underline{m}^{\prime}\right) \frac{}{m_{0}^{\prime} L_{0}^{\prime}} m_{0}^{\prime \prime} L_{0}^{\prime \prime} L^{\prime \prime}\left(\underline{m}^{\prime \prime}\right)$ de deux multi-entrelacs est définie si et seulement si les multiplicités $\underline{m}^{\prime} \in \operatorname{Hom}\left(H_{1} X^{\prime}, \mathbb{Z}\right)$ et $\underline{m}^{\prime \prime} \in \operatorname{Hom}\left(H_{1} X^{\prime \prime}, \mathbb{Z}\right)$ coïncident sur l'homologie du tore de recollement $\partial \mathcal{N}\left(L_{0}^{\prime}\right)=\partial \mathcal{N}\left(L_{0}^{\prime \prime}\right) \subset \Sigma$. On aboutit aux équations

$$
m_{0}^{\prime}=\sum_{j=1}^{r} m_{j}^{\prime \prime} \ell k\left(L_{0}^{\prime \prime}, L_{j}^{\prime \prime}\right) \quad \text { et } \quad m_{0}^{\prime \prime}=\sum_{i=1}^{n} m_{i}^{\prime} \ell k\left(L_{0}^{\prime}, L_{i}^{\prime}\right) .
$$

Un entrelacs simple est un entrelacs irréductible $L$ tel que tout tore incompressible dans l'extérieur de $L$ est parallèle au bord. Un entrelacs seifertique est un entrelacs dont l'extérieur admet un feuilletage de Seifert. Par le théorème de décomposition de Jaco-Shalen (voir [3]), tout entrelacs irréductible $L$ dans une 3-sphère d'homologie peut être exprimé comme l'épissure d'un nombre fini d'entrelacs simples ou seifertiques, et il existe une unique façon minimale de le faire. On parle de la décomposition de Jaco-Shalen de L, les composantes de cette décomposition étant les éléments d'épissure de L. Un entrelacs de Waldhausen est un entrelacs dont tous les éléments d'épissure sont des entrelacs seifertiques.

Exemple 2. - Les entrelacs de Waldhausen dans $S^{3}$ sont exactement les entrelacs toriques itérés, c'està-dire, les entrelacs constructibles par cablage à partir d'entrelacs triviaux.

Eisenbud et Neumann ont donné dans [2] une classification des multi-entrelacs de Waldhausen au moyen de diagrammes d'épissure, définis comme suit. Tout multi-entrelacs seifertique peut être représenté par un diagramme de la forme du premier dessin de la Figure 1 Les $\alpha_{i}$ sont les poids associés aux feuilles exceptionnelles du feuilletage de Seifert, les pointes de flèche symbolisent les composantes de l'entrelacs, et le 


\section{L'homologie de Novikov des entrelacs de Waldhausen}

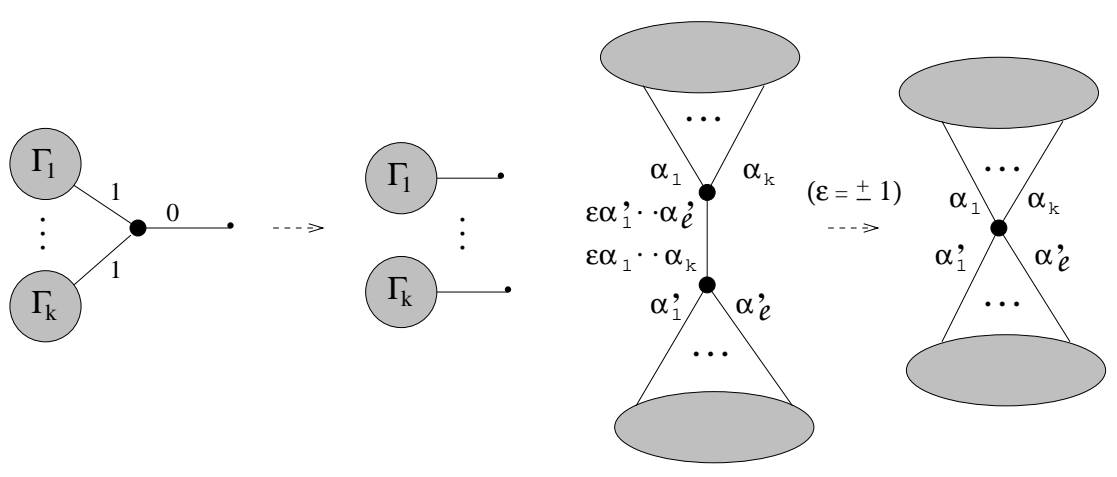

Figure 2: Reductions de diagrammes/Reduction of splice diagrams

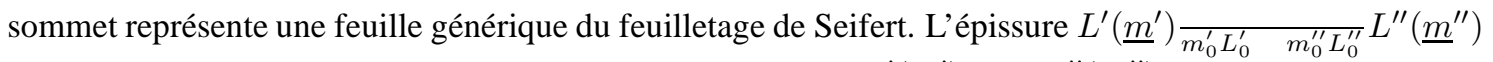
de deux multi-entrelacs est symbolisée par les diagrammes de $L^{\prime}\left(\underline{m}^{\prime}\right)$ et de $L^{\prime \prime}\left(\underline{m}^{\prime \prime}\right)$ reliés le long des arêtes correspondant à $L_{0}^{\prime}$ et à $L_{0}^{\prime \prime}$ (voir Figure 1). L'opération inverse sur un diagramme consiste à couper une arête reliant deux sommets et à ajouter deux pointes de flèches munies des multiplicités données en $(*)$; on parle de décomposition du diagramme. Enfin, l'union disjointe de diagrammes représente la somme disjointe des multi-entrelacs. Bien entendu, deux diagrammes d'épissure différents peuvent représenter le même multi-entrelacs. Par exemple, les réductions illustrées en Figure 2 ne changent pas le multi-entrelacs représenté. Si aucune de ces deux réductions ne peut être effectuée, on dira que le diagramme est minimal.

Les diagrammes d'épissure sont parfaitement adaptés au calcul des coefficients d'enlacement. Etant donnés deux sommets ou pointes de flèche $v$ et $w$, le coefficient d'enlacement $\ell(v, w)$ des courbes correspondantes (feuille générique de Seifert ou composante de l'entrelacs) est donné par la formule suivante: soit $\sigma(v, w)$ la géodésique du diagramme reliant $v$ et $w$ ( $v$ et $w$ inclus); alors, $\ell(v, w)$ est le produit de tous les poids des arêtes adjacentes à $\sigma(v, w)$ mais pas sur $\sigma(v, w)$.

De plus, il est très facile de lire sur son diagramme minimal si un multi-entrelacs est fibré ou non. Un multi-entrelacs est fibré si et seulement s'il est irréductible et chacun de ses éléments d'épissure est fibré. Un multi-entrelacs seifertique est fibré si et seulement si son coefficient d'enlacement avec une feuille générique est non-nul. Ainsi, le multi-entrelacs représenté par le premier diagramme (minimal) de la Figure 1 est fibré si et seulement si

$$
\sum_{i=1}^{n} m_{i} \alpha_{1} \cdots \widehat{\alpha}_{i} \cdots \alpha_{k} \neq 0 .
$$

Par conséquent, les entrelacs de Waldhausen irréductibles sont génériquement fibrés. En particulier, leur homologie de Novikov est génériquement triviale.

Nous sommes enfin en mesure d'énoncer la formule pour l'homologie de Novikov d'un entrelacs de Waldhausen.

\section{Résultats}

Soit $L=L_{1} \cup \cdots \cup L_{n}$ un entrelacs de Waldhausen donné par un diagramme d'épissure minimal $\Gamma$, et soit $\underline{m} \in \mathbb{Z}^{n}-\{0\}$. Il est facile de vérifier que les modules d'Alexander de $\left(L^{\prime}\left(\underline{m}^{\prime}\right) \cup 0 L_{0}^{\prime}\right)-0 L_{0}^{\prime \prime}$ et de $L^{\prime}\left(\underline{m}^{\prime}\right)$ sont égaux; il en va donc de même pour les modules de Novikov, et nous allons supposer que ces simplifications ont été faites dans $\Gamma(\underline{m})$.

Chaque sommet de $\Gamma(\underline{m})$ correspond à un multi-entrelacs seifertique, fibré ou non-fibré; on parlera de sommet fibré ou non-fibré. Par ailleurs, on dira qu'un sommet est un 0-sommet si toutes les multiplicités du multi-entrelacs seifertique correspondant sont nulles. Soit $\Gamma^{\prime}\left(\underline{m}^{\prime}\right)$ le sous-diagramme de $\Gamma(\underline{m})$ obtenu de la 


\section{David Cimasoni}

façon suivante: on décompose $\Gamma(\underline{m})$ le long des arêtes reliant un sommet fibré et un sommet non-fibré, et on efface toutes les composantes connexes correspondant à des multi-entrelacs fibrés. Soient $c$ le nombre de composantes connexes de $\Gamma, r$ le nombre de composantes connexes de $\Gamma^{\prime}\left(\underline{m}^{\prime}\right), n$ le nombre de flèches de $\Gamma^{\prime}\left(\underline{m}^{\prime}\right)$ et $k$ le nombre de ses sommets. Pour chaque sommet $v$ de $\Gamma^{\prime}\left(\underline{m}^{\prime}\right)$, notons encore $\alpha(v)$ le produit des poids des flèches de $\Gamma^{\prime}\left(\underline{m}^{\prime}\right)$ adjacentes à $v$.

THÉORÈME 3.1. - L'homologie de Novikov du multi-entrelacs $L(\underline{m})$ est la somme directe

- d'un facteur libre de rang $n+c-r-k-1$, et

- d'un facteur présenté par la $(k \times k)$-matrice $P=\left(p_{v w}\right)$, avec $v$ et $w$ parcourant l'ensemble des sommets non-fibrés, où $p_{v w}=\frac{\ell(v, w)}{\alpha(w)}$ si $v$ et $w$ appartiennent à la même composante connexe de $\Gamma^{\prime}\left(\underline{m}^{\prime}\right)$ et $v$ n'est pas un 0-sommet, et $p_{v w}=0$ sinon.

Donnons un très bref aperçu des techniques utilisées. La première étape consiste à adapter les notions classiques de surfaces et de formes de Seifert aux multi-entrelacs. Ces formes de Seifert "généralisées" permettent de calculer le module d'Alexander des multi-entrelacs seifertiques non-fibrés. Ensuite, il s'agit de comprendre l'effet de l'épissure sur les modules considérés. En travaillant sur $\mathbb{Z}\left[t, t^{-1}\right]$ (i.e: en étudiant le module d'Alexander), il est extrêmement difficile de donner une formule close. En revanche, le passage à l'homologie de Novikov (en d'autres termes, la tensorisation par $\mathbb{Z} \llbracket t \rrbracket\left[t^{-1}\right]$ ) permet de simplifier considérablement les calculs (voir [1] pour les détails).

Corollaire 3.2. - Soit $L=L_{1} \cup \cdots \cup L_{n}$ un entrelacs de Waldhausen. Il existe un nombre fini d'hyperplans dans $\mathbb{Z}^{n}$, définis par des équations linéaires homogènes à coefficients entiers, tels que $\widehat{H}_{L}(\underline{m})$ est constant sur chaque strate de la "stratification" de $\mathbb{Z}^{n}$ par ces hyperplans.

Voir [5], Théorème 3, p. 529 pour un résultat analogue dans un autre contexte.

COROLlAIRE 3.3. - Soit un entrelacs de Waldhausen représenté par un diagramme d'épissure minimal $\grave{a} k$ sommets et c composantes connexes. Alors, le nombre de modules de Novikov est borné par $3^{k}-2(k-c)$.

Comme nous l'a fait remarquer A. Pazhitnov, ce phénomène de finitude du nombre de modules semble exceptionnel en homologie de Novikov.

Exemple 3. - Notons $\hat{\Lambda}$ l'anneau de Novikov $\mathbb{Z} \llbracket t \rrbracket\left[t^{-1}\right]$. L'entrelacs seifertique L correspondant au diagramme minimal donné en Figure पsatisfait

$$
\widehat{H}_{L}(\underline{m})= \begin{cases}0 & \text { si } \underline{m} \in \mathbb{Z}^{n}-V, \\ \hat{\Lambda} /\left(\alpha_{n+1} \cdots \alpha_{k}\right) \oplus \hat{\Lambda}^{n-2} & \text { si } \underline{m} \in V-\{0\}, \\ \hat{\Lambda}^{n} & \text { si } \underline{m}=0,\end{cases}
$$

où $V=\left\{\left(m_{1}, \ldots, m_{n}\right) \in \mathbb{Z}^{n} \mid \sum_{i=1}^{n} m_{i} \alpha_{1} \cdots \widehat{\alpha}_{i} \cdots \alpha_{k}=0\right\}$.

Remerciements. Je souhaite remercier vivement Claude Weber, Andrei Pazhitnov et Mathieu Baillif pour leur aide. C'est également un plaisir de témoigner toute ma gratitude à Jerry Levine pour son accueil et sa disponibilité à l'Université de Brandeis.

\section{Références bibliographiques}

[1] Cimasoni D., Thèse de Doctorat (en préparation), Université de Genève.

[2] Eisenbud D. et Neumann W.D., Three-dimensional link theory and invariants of plane curve singularities, Annals of Mathematics Studies, 110. Princeton University Press, Princeton, N.J., 1985.

[3] Jaco W.H. et Shalen P.B., Seifert fibered spaces in 3-manifolds, Mem. Amer. Math. Soc. 21 (1979), no. 220.

[4] Novikov S.P., Multivalued functions and functionals. An analogue of the Morse theory, Soviet Math. Dokl. 24 (1981), no. 2, p. 222-226.

[5] Pazhitnov A.V., Exactness of Novikov-type inequalities for the case $\pi_{1} M=Z^{m}$ and for Morse forms whose cohomology classes are in general position, Soviet Math. Dokl. 39 (1989), no. 3, p. 528-532. 